\title{
Physicochemical Stages of Evolution: Ring-Like Structures in the Universe
}

\author{
Georgi P. Gladyshev'1,2 \\ ${ }^{1}$ International Academy of Creative Endeavors, Moscow, Russia \\ ${ }^{2}$ N. N. Semenov Institute of Chemical Physics, Russian Academy of Sciences, Moscow, Russia \\ Email: academy@creatacad.org
}

Received 8 April 2015; accepted 16 May 2015; published 18 May 2015

Copyright (C) 2015 by author and Scientific Research Publishing Inc.

This work is licensed under the Creative Commons Attribution International License (CC BY). http://creativecommons.org/licenses/by/4.0/

c) (i) Open Access

\section{Abstract}

This short digest is devoted to the mechanism of ring formation during the origination and evolution of planetary and satellite systems in the Universe. The appearance of these structures can be traced back to the phenomenon of spatially periodic condensation which can be observed on the Earth. The author actually posited the existence of exoplanetary systems and the cosmic scale of this phenomenon. It was accurately predicted that Uranus, Neptune and other heavenly bodies have rings. The suggested general mechanism rationalized the Titius-Bode law which, while not being a precise law, often accurately describes the tendency towards varying distances between planets (and their moons) and central bodies. The possibility of this law manifestation in exoplanetary systems had been predicted by the author long before their discovery. Many exoplanetary systems have been discovered by now and there is some evidence corroborating the mechanism of spatially periodic condensation involved in the formation of ring-like structures in these systems. The author's hypothesis is now becoming a theory or a fact. It appears that we are now witnessing the dawn of a new extensive cosmology, taking into account general physicochemical mechanisms of space object formation.

\section{Keywords}

Cosmology, Evolution, Exoplanetary Systems, Liesegang Phenomenon, Solar System, Titius-Bode Law

The purpose of the present digest is drawing the attention of cosmology researchers to the general mechanism of origination of ring-like structures emerging during the formation of planets, their satellites and other bodies in the Universe.

In February 1977 the author published his work titled On the Question of the Role of Physicochemical 
Processes in Planetary Systems Formation [1]. It was postulated in this work and the identical article The Physicochemical Mechanism of Planetary System Formation [2]: "The planets and their satellites are formed in accordance with similar mechanisms as a result of spatially periodic condensation of gaseous matter during the formation of the central body. Using the diffusion theory, one can calculate the age of the planets and explain the nature of the Titius-Bode law.”

The above-mentioned works [1]-[3] focused on the model of spatially periodic condensation during the formation of planetary and satellite systems which had not yet been discovered back then. A similar condensation phenomenon had earlier been observed on the Earth by Friedlieb Ferdinand Runge, Raphael Eduard Liesegang and other researchers. Friedrich Wilhelm Ostwald christened this process as "the Liesegang phenomenon" [4].

The author of the present work postulated [1] [2] that the Liesegang phenomenon model could be applied on the planetary scale. While devising this hypothesis, the author actually used the principle of reductionism and spread the concept "As Above So Below" [5] to hierarchical structures of the Universe [1]-[3] [6] [7].

The aforementioned simplified model draws on the general idea of protostar formation from interstellar gasand-dust nebulas in galaxies. In the course of evolution protoplanetary rings take shape in the "protostar - its primary accretion disk" system, with planets later formed out of these rings. Originating in the course of planet formation are primary satellite accretion disks followed by rings that give rise to planet satellites (moons). Therefore the model implies the existence of the general standard mechanism of planet birth around stars and satellite birth around planets.

It is actually postulated that planetary and satellite systems come into existence through a standard mechanism in objects consisting of the central body (protostar, protoplanet, protosatellite or some other central body) and gas-and-dust disk surrounding it.

When the central body grows big enough, it can heat up by virtue of gravitational contraction, the energy of radioactive decay, nuclear bombardment reactions or other physical phenomena. As time goes by, the central body takes on a spherical shape. Later on this revolving effervescent central body starts releasing ("emitting") gaseous (sometimes also dust-like) matter into the disk encircling it. The released matter is transported by means of diffusion or other mass-transfer mechanisms into the disk's inner areas. A quite possible next stage is the formation of solid precipitation rings from supersaturated gas. Two phase formation mechanisms can be distinguished.

1) In the course of chemical reactions in the protostar or protoplanet disk spatially periodic structures are formed, following the "Liesegang phenomenon" pattern. This phenomenon is accompanied by the formation of tenuous rings which give rise to planets and their satellites. For instance, the graphic illustration of the reaction between $\mathrm{NH}_{3}$ and $\mathrm{HCl}$ that yields the precipitation $\mathrm{NH}_{4} \mathrm{Cl}$ in the gas phase was presented in laboratory setting by V.N. Gorshenev and the author of this article [7] [8]. Normally studied in laboratory setting are precipitation reactions from the interaction of two individual substances. However, in real natural systems different chemical agents are often accumulated in "oversaturated areas". This may trigger the formation of "randomly situated" mixed precipitation comprised of various compounds. Thus, the formation of adjacent planetary rings from mixtures of various compounds released from the protoplanet is accompanied by emergence of the ring structure which does not have homogeneous spatial periodicity. The gases ejected by the planet may entrain small solid particles accumulated in the rings. Moreover, on account of the rapid evolution, the chemical makeup of the rings may undergo significant changes. The separation of chemical agents and isotopes is also possible in the course of diffusion (mass transfer) of the matter released from the central body into the adjacent disk where temperature and pressure gradients exist. This complex substance separation effect can apparently be described as "mixed chromatography from the oversaturated state."

2) In the process of gaseous individual matter release (emission) from the central body into empty "inert" space the formation of the solid phase of this matter out of oversaturated or overcooled condition can be observed. Similar phenomena take place in the process of formation of some clouds (e.g. cirrus uncinus clouds) in the planet atmosphere and the emergence of ring-like structures near comet nuclei (e.g. Comet Hale-Bopp, 1997). In some cases nearly homogeneous in terms of makeup adjacent rings can be observed around central bodies. In such systems "regular" structures are to be formed.

It is necessary to note that the origination of rings in planetary systems can be observed in revolving disks. This is why two-dimensional precipitation structures take the form of rings. Yet when mass transfer occurs inside the (volume) of the bodies, where temperature gradients and limited convection exist, 3D spatially periodic structures may occur. It is known that such structures can exist inside planets and their atmospheres in "noncon- 
vective” zones. As was earlier indicated, occasionally arising are lens-like clouds whose 3D structure is linked with temperature gradients defined by the form of the earth surface. Existing here is complete analogy between 3D structures of minerals, such as agates, and 3D large-scale structures emerging in heavenly bodies and their atmospheres. Interestingly enough, the oversaturation phenomenon is used in Wilson chamber and other devices.

Thus both in the terrestrial and extraterrestrial environment there exist various conditions and systems, where the formation of the above-stated spatially periodic structures is possible. It's clear that the origination of such structures has some specifics in different cases, so it's hardly advisable to create a universal detailed model of this phenomenon. However, there is one thing in common in all of the above-described processes: the existence of critical values. Their reaching is accompanied by the phenomenon of spatially periodic condensation with the formation of both regular and irregular structures. Spatially periodic structures and objects of their transformation in the Universe constantly evolve under the impact of various physical forces, which results in the alteration of these structures and mutual alignment of rings and heavenly bodies in astrophysical objects.

Apart from critical parameters (e.g. saturation values), in all cases of spatially periodic condensation from the gaseous phase we apparently see another commonality: the processes of spatially periodic structure formation are usually close to the state of equilibrium. The processes can be understood within the framework of dynamic thermodynamics of close to equilibrium systems [7]. In describing these phenomena one can dispense with the idea of dissipative structures and "thermodynamics" of far from equilibrium systems.

The author reviewed some specifics of the proposed general mechanism of planetary system formation in a number of his works [1]-[3] [6] and in the monograph [7]. Regrettably, this book was never translated into English because of competing interests and prevalent corruption. However, two years later the author, along with D.H. Kitaeva, presented some of the materials from the monograph as a digest in English [9]. Thus the English audience better familiarized themselves with works in this area.

Now we have more evidence that many astronomical objects have rings. Moreover it becomes obvious that planets and their satellites originate from rings emerging at the initial and subsequent stages of central body formation [10] [11]. This means that not all bodies in planetary systems are formed almost "simultaneously", as the prevalent theories imply. Rather planets and their satellites evolve during a relatively long period of time. Planets and their satellites closest to central bodies are older than planets and their satellites moved farther away from central bodies. Relatively young planets like Saturn, Uranus, Neptune, Pluto and others have retained visible evolving rings, some of these lying in the Roche zones. As per the calculations presented in the works [1]-[3] [5]-[9], the age of Saturn is roughly 4.3 bn years, Uranus - 3.6 bn years, Neptune-2.3 bn years. The tentative age of Pluto is about 0.6 bn years. It should be noted that the ages of Mercury, Venus, Earth and Mars are comparable and close to 4.5 bn years. Jupiter's age is a bit smaller-roughly 4.4 bn years. These figures can be updated if the simple model is further elaborated and the results of new observations are taken into account.

Revolving spherical heavenly bodies normally have rings. However, it should be borne in mind that as massive bodies are formed, gravitational effects are activated from the rings and the Titius-Bode law can be notably distorted. These and some of the above-mentioned physicochemical reasons show that the Titius-Bode law (that generally works, as the author posited, for all planetary systems in the Universe) should not be regarded as the accurate or precise law. Nevertheless, this law ("the law of receding rings as a trend") often reveals the mutual alignment of ring structures and hence the orbits of the bodies originating from these structures in the process of forming stars, planets, satellites and other heavenly bodies in the Universe. In some cases the law helps finding exoplanets and exosatellites in exoplanetary systems.

There are grounds to believe that young spherical heavenly bodies may have some rings or ring structures which fully or partly transform in evolving "planetary-type” systems. The presented idealized model may certainly be amplified and further elaborated as far as reasonably advisable. For instance, the model may take into account transformations of primary disks in the process of evolution of planetary, satellite and other similar systems. Perhaps it is necessary to consider the specifics of spatially periodic condensation mechanisms in different areas of primary (proto) disks - the areas having different chemical compositions, temperatures, pressures and other physicochemical characteristics. In accurate calculations it is necessary to take into consideration the size of turbulence zones adjacent to the surface of central bodies. Within these zones the transfer of matter does not follow the diffusion mechanism, which is a basic assumption of the simple inaccurate model [1]-[3].

One of the interesting questions regarding the origin of ring-like galaxies and other mega structures remains open - is it possible to create a spatially periodic model of hydrogen formation from the "oversaturated state" of 
elementary particles in the evolving Universe?

Despite the huge diversity of evolutionary transformations in space, it is obvious that spatially periodic condensation from the gaseous phase during the formation of planetary systems is an important stage in the evolution of matter in many domains of the Universe. It cannot be altogether excluded that the hope of the author of As Above So Below [5] will come true: "Perhaps, in the longer term, it may be possible to develop a synthesis cosmology based upon the intriguing ideas of Georgi Gladyshev, Timo Niroma, Johannes Kepler and Robert James Moon.”

\section{References}

[1] Gladyshev, G.P. (1977) On the Question of the Role of Physicochemical Processes in Planetary Systems Formation, Preprint, 1-8. Institute of Chemical Physics, U.S.S.R. Academy of Sciences, Chernogolovka, U.S.S.R, 28 February. http://creatacad.org/?id=22\&lng=eng http://creatacad.org/?id=24\&lng=eng

[2] Gladyshev, G.P. (1978) The Physicochemical Mechanism of the Formation of Planetary System. The Moon and Planets, 18, 217-221. http://link.springer.com/article/10.1007/BF00896744 http://dx.doi.org/10.1007/bf00896744

[3] Gladyshev, G.P. (1978) On the Mechanism of Reaction in Rarefied Gas: Processes in the Solar System. The Moon and Planets, 19, 89-98. http://link.springer.com/article/10.1007\%2FBF00898167\#page-1 http://dx.doi.org/10.1007/BF00898167

[4] Coyle, J. (2013) Liesegang Rings: 1-The Liesegang Phenomenon. https://malagabay.wordpress.com/2013/10/27/liesegang-rings-1-the-liesegang-phenomenon/https://malagabay.wordpre ss.com/category/liesegang-rings/

[5] Niroma, T. (2014) As Above So Below-Georgi Gladyshev. https://malagabay.wordpress.com/2014/05/05/as-above-so-below-georgi-gladyshev/

[6] Gladyshev, G.P. and Budtov, V.P. (1981) The Solar System Evolution. The Moon and Planets, 25, 413-425. http://link.springer.com/article/10.1007\%2FBF00919076 http://dx.doi.org/10.1007/bf00919076

[7] Gladyshev, G.P. (1988) Thermodynamics and Macrokinetics of Natural Hierarchical Processes. Nauka Publ., Moscow, 288 pp. (In Russian).

[8] Gorshenev, V.N. and Gladyshev, G.P. (1981) Using the Counter Diffusion Method to Determine Critical Concentrations of Precipitating Components in Periodic Reactions. The Journal of Physics and Chemistry, 55, 2897-2903.

[9] Gladyshev, G.P. and Kitaeva, D.Kh. (1990) Some Problems in Chemical Evolution of Matter in Space. Zhurnal Vses. Khim. Ob-va im. D.I. Mendeleeva, Vol. 35, No. 5, pp. 625-631, UDC 523-52:523.4-36. http://creatacad.org/?id=23\&lng=eng

[10] Crida, A. and Charnoz, S. (2012) Formation of Regular Satellites from Ancient Massive Rings in the Solar System. Science, 338, 1196-1199. http://www.sciencemag.org/content/338/6111/1196 http://dx.doi.org/10.1126/science.1226477

[11] Birth of Planets Revealed in Astonishing Detail in ALMA’s “Best Image Ever” Nation Radio Asronomy Observatory. For Release: 7 a.m. E.S.T. Thursday, November 6, 2014. 